\title{
Hombres que ejercen violencia hacia la (ex) pareja mujer: cambios y tensiones.
}

\section{Men who exert violence towards their (ex) female partner: changes and tensions}

Nicol Astorga*

Alex Valdivia

\begin{abstract}
Resumen
El presente artículo da cuenta de la sistematización de un proceso reeducativo dirigido a hombres en un programa estatal. El objetivo fue identificar los elementos fundamentales de la intervención que vivieron 11 hombres en el Centro de Hombres por una vida sin violencia de La Serena, Región de Coquimbo, Chile, desde julio 2017 a marzo 2019. Se describen los aprendizajes y cambios que éstos reconocieron, identificando los facilitadores y obstaculizadores del proceso grupal, y finalmente, discutiendo los discursos de los mismos hombres y sus parejas o ex parejas mujeres. Se realiza un análisis documental de los sustentos teóricos del programa, análisis de los registros de audio y escritos de intervenciones individuales y grupales, y se contrasta esta información con los reportes de las mujeres parejas y/o ex parejas de ellos. Los resultados dan cuenta que en este proceso los hombres por primera vez cuestionan su biografía desde una perspectiva de género, relacionándola con la cultura patriarcal de la sociedad chilena; desarrollan conductas y discursos empáticos hacia mujeres y otras personas; logran diferenciar entre respeto y miedo. Incorporan elementos de escucha activa y señalan mejorar su comunicación a nivel conyugal y parental. En cuanto a facilitadores, los hombres identifican el respeto y la participación en espacio grupal, así como la acogida y escucha activa hacia ellos. Respecto a los obstaculizadores del proceso, identifican la vergüenza "social" por los actos cometidos y también, los ambientes machistas en los que se desenvuelven cotidianamente.
\end{abstract}

Palabras clave: Violencia, masculinidades, género, educación, facilitadores, obstaculizadores, aprendizajes, cambio.

\footnotetext{
"Trabajadora Social, Universidad Tecnológica de Chile. Corporación Municipal de Educación Gabriel González Videla. nicol.astorga@gmail.com

**Psicólogo, Universidad de La Serena. Centro de Hombres por una Vida Sin Violencia. alex.valdiviaa@gmail.com
} 


\begin{abstract}
This article reports on the systematization of a re-educational process directed at men in a state program. The objective was to identify the basic elements of the intervention that 11 men lived in the Men's Center for a life without violence in La Serena, Coquimbo Region, Chile, from July 2017 to March 2019. The learnings and changes that they recognized are described, identifying the facilitators and obstacles to the group process, and finally, discussing the discourses of the same men and their partners or former female partners. A documentary analysis of the theoretical support of the program, analysis of the audio and written records of individual and group interventions is carried out, and this information is contrasted with the reports of the female partners and / or former partners of them.

The results show that in this process men for the first time question their biography from a gender perspective, relating it to the patriarchal culture of Chilean society; develop empathetic behaviors and discourses towards women and other people; they manage to differentiate between respect and fear. They incorporate elements of active listening and point to improve their communication at the marital and parental level. As for facilitators, men identify respect and participation in group space as well as the reception and the active listening to them. Meanwhile, in the obstacles, they identify the "social shame" because of the violence committed, and, also, the sexist social spaces where they develop daily.
\end{abstract}

Keywords: Violence, masculinities, gender, education, facilitators, obstacles, learning, change.

Fecha de recepción: Diciembre 2019

Fecha de aprobación: Junio 2020

\title{
Introducción
}

La presente sistematización da cuenta de un proceso psico-socioeducativo con hombres que ejercieron violencia hacia su pareja o ex pareja mujer, que participaron del Programa Centro de Hombres por una vida sin violencia. Quienes realizamos este ejercicio, cumplíamos las funciones de Trabajadora Social y Psicólogo, como dupla psicosocial. Desde el rol de facilitadores de estos grupos, nos surgió el interés de sistematizar el proceso vivido por los hombres, pensando en que, a diferencia de años atrás, no habían coincidido tantos varones en el mismo período, junto con un escaso registros de trabajos de este tipo en Latinoamérica.

Se presenta la experiencia de trabajo con hombres, luego de ser parte de un proceso de intervención, primero individual, y luego grupal, dentro del dispositivo estatal Centro de Hombres por una vida sin violencia, dependiente de Servicio Nacional de la 
Mujer y Equidad de Género de la Región de Coquimbo, Chile durante los años 20172018.

Para entender por qué y para qué se trabaja con hombres es necesario mencionar la misión del Servicio Nacional de la Mujer y Equidad de Género (desde ahora SERNAMEG) como una institución estatal que busca: "promover en la sociedad la igualdad, autonomía, equidad, no discriminación y una vida libre de violencia para las mujeres en toda su diversidad y la implementación de políticas, planes y programas que transversalicen la equidad de género en el Estado" (SERNAMEG, 2019). Desde allí, podemos entender que SERNAMEG, mediante sus programas desplegados a nivel nacional, ha contribuido a reconocer la violencia como un problema con alta prevalencia en la vida de las mujeres. En esta línea, a lo largo de sus años de existencia como institución (desde 1991), ha brindado atención y protección a mujeres con sus diferentes modelos o dispositivos, abarcando las dimensiones psicológica, social y jurídica. Sin embargo, a partir de estadísticas internas del mismo servicio, se evidenció que entregar atención psico-sociojurídica a las mujeres, y no hacerlo con los hombres, resultaba ineficiente en la detención de la violencia, ya que alrededor de un $64 \%$ de las mujeres que acudían a Centros de la Mujer (dispositivos de atención a mujeres que viven violencia por parte de su pareja o ex pareja), reportaban seguir viviendo o manteniendo una relación con el hombre que las maltrataba. Por lo tanto, la atención por sí sola a las mujeres que viven violencia, no era suficiente.

Los Centros de hombres nacen el año 2011 como parte de la estrategia de SERNAMEG para "avanzar en poner término a la violencia contra las mujeres en las relaciones íntimas. Se trata de una intervención que busca disminuir los niveles de violencia hacia las mujeres y favorecer su seguridad y protección ante nuevos hechos de violencia, siendo una intervención con HEVPA (Hombres que Ejercen Violencia de Pareja) dirigida a mejorar e impactar positivamente en la calidad de vida de las mujeres, restituyendo sus derechos humanos y libertades fundamentales que han sido vulneradas por los malos tratos." (SERNAMEG 2019).

Entonces, nos preguntamos ¿Qué tanto los hombres logran cambiar en un proceso como éste?, y ¿Cuáles son los elementos que permiten o dificultan estos cambios? Desde que nos interesamos en el proceso grupal de re-educación en el que nos vimos inmersos, decidimos enfocarnos en los aspectos que creíamos podrían ser los ejes claves a considerar para visibilizar las vivencias y lecciones que podríamos sacar de esta experiencia. Nos planteamos como objetivo general identificar elementos fundamentales en el proceso de re-educación con hombres que ejercieron violencia hacia sus parejas o ex parejas mujeres, usuarios de HEVPA, La Serena. En términos específicos, nos propusimos describir los aprendizajes y cambios que los hombres reconocieron durante el proceso de reeducación grupal; reconocerlos facilitadores y obstaculizadores del proceso grupal de psico-socioeducación en hombres de primer y segundo nivel, y discutir los aprendizajes reconocidos por los hombres en el proceso de reeducación grupal.

Creemos en la importancia de visibilizar la experiencia vivida, con el fin de difundir el trabajo realizado con hombres, destacar los relatos y encuentros, así como las 
lecciones y aprendizajes que podemos identificar una vez concluido este trabajo de sistematización.

Se trabajó en base al modelo Alforja de Oscar Jara, para buscar una comprensión profunda de las experiencias a fin de mejorar la práctica, desde una interpretación crítica de las experiencias que vivimos en el proceso grupal. El tipo de muestreo es intencional, ya que los hombres elegidos fueron seleccionados por haber sido participantes activos de primer y segundo nivel en los espacios grupales de HEVPA entre 2017-2019. Ambos grupos fueron seleccionados por la motivación y adherencia que presentaban al centro. La unidad de análisis fueron 11 hombres usuarios de HEVPA derivados por Tribunales de Familia y Tribunales de Garantía, residentes de la Región de Coquimbo, quienes asistieron a un proceso psico-socioeducativo de reeducación en violencia de género, en Centro de Hombres Región de Coquimbo entre los años 2017 y 2018. El promedio de edad era de 41 años, seis de ellos con nivel educacional universitario, tres de ellos con una educación media completa, y los dos restantes con estudios secundarios de nivel técnico completo. En cuanto a su ocupación, dos de ellos eran arquitectos, dos eran pedagogos, un administrador de empresas, un analista de sistemas, un jubilado del ejército, un mecánico, un chofer, un operador de maquinaria pesada y un ingeniero. El promedio de tiempo que permanecieron en el proceso en HEVPA fue de 18 meses, siendo 24 meses el de mayor permanencia y 12 meses el que permaneció menos. Cuatro de estos hombres mantuvieron su relación de pareja, mientras que 7 de ellos no volvió a retomar su relación de pareja. Para participar del proceso terapéutico y de la sistematización de la experiencia que se expone en este artículo, cada varón firmó un consentimiento que autoriza el uso posterior de la información producida.

Para registrar las vivencias, se utilizaron grabaciones en audio de las sesiones de grupo focal, correspondientes a la sesión de cierre grupal de primer y segundo nivel. En cuanto al análisis documental, se realizó una revisión crítica de los registros de atenciones individuales y grupales de los hombres. Además, se realizó una revisión documental de los registros de evaluación de cada varón en el proceso grupal, reporte de seguimiento a las mujeres parejas o ex parejas de ellos, cartas escritas por ellos a su pareja o ex pareja al ingreso al centro y al egreso de éste, papelógrafos de las sesiones grupales y fotografías de las sesiones.

\section{Aprendizajes y cambios}

La historia de Chile ha sido moldeada para que minimicemos actos de violencia estatal, colonial y militar (golpes de estado, guerras civiles y genocidio indígena), en lo que se podría denominar como una cultura de violencias sociales. En este escenario, al momento del ingreso y trabajo inicial con los hombres, a medida que las sesiones iban avanzando, reconocían que nunca en su vida escucharon sobre otras alternativas diferentes a la violencia para lograr resolver un conflicto.

"Es una actitud aprendida, que se normaliza, que muchas veces tiene que ver con miedo propio que se disfraza bajo esta actitud dominante sobre estos" (Registro de atención individual). 
Algunos teóricos de género y masculinidades (Connell, 2003), han señalado que mediante la educación y construcción social que recibimos desde pequeños, internalizamos y promovimos una identidad masculina hegemónica, un modelo, una forma de ser hombre que no sólo busca ejercer violencia y predominancia sobre quienes no cumplen con ese molde, sino también legitima el poder masculino en las organizaciones sociales, las instituciones y la propia cultura. Cada hombre tiene definiciones de acuerdo con las particularidades de sus propias culturas, razón por la cual el patriarcado y la heteronorma no permanecen estáticas, sino que, por el contrario, se moldean y ajustan a cada realidad.

Al ingreso de los hombres a la fase grupal resultó fundamental comenzar a trabajar desde los aspectos más generales de la violencia, desde el reconocimiento de ésta y los tipos de manifestación. Comienzan a conocer conceptos y temáticas que nunca habían tenido la oportunidad de escuchar y cuestionar. Logran tipificar las violencias, como ejercicios de poder y control de sus contextos, logran comprender que las violencias que decidieron ejercer, tuvieron consecuencias. Según Kimmel (2000), esta masculinidad debe demostrarse constantemente, ya que aprendemos que, de lo contrario, podemos perder el control y el poder ejercidos sobre otras y otros, y puede llegar a volverse en contra.

Cabe preguntarse: si estas son las consecuencias propias de un modelo aprendido y transmitido, ¿existen otras formas de aprender a ser hombre?, ¿qué tipo de educación se requiere para tomar consciencia de esta forma de ser hombre en la que hemos sido instruidos, y considerar otras formas de vivir la masculinidad?

El trabajar desde la educación popular es situarse, no desde el rol de experto/a, sino verse de igual manera como un ser que se nutre de los y las demás, que están en constante aprendizaje, por lo que nosotros/as también comentábamos y participábamos de todas las actividades.

Cuando los hombres se encontraban en el siguiente nivel de la intervención (segunda etapa de temáticas por abordar, que tiene como duración cuatro meses, al menos), recién lograban manifestar conductas vinculadas a la empatía con quienes habían violentado en el pasado. Previo a esto, es decir, durante el primer nivel (primera etapa de temáticas, de tres meses de duración), desarrollaban el reconocimiento de sí mismos como hombres que habían ejercido violencia y su responsabilización.

"el ponerse en el lugar de otro, el pensar que me pasaría a mi si estuviera ahí, y pedir las disculpas cuando uno se equivoca, aceptar la responsabilidad", "aceptar la culpa, pedir perdón, disculpas", "la igualdad con otras personas, no por ser hombres vamos a ser más" (Audios de sesión grupal).

Los hombres en proceso señalaban haber aprendido en esta experiencia que la forma en que uno se comunica, es esencial al momento de resolver conflictos. Señalaban que terminaban por replicarla mucha de la violencia de la misma manera que sus padres o abuelos, y que la violencia que habían repetido, tenían, a su vez, la oportunidad de no volver a replicarla. 
Los hombres logran visualizar particularidades de su vida personal en torno a los ejercicios de poder:

"A no abusar como hombres, el respeto, el control y a compartir más porque a veces uno en cuanto a aprendizajes de las labores de la casa y ayudar para compartir más y uno es más flojo y no había cosas que eran necesarias de hacer y uno no lo hacía y ahora uno lo hace." (Audios de sesión grupal).

De cierta manera, el centro de hombres era un espacio en donde podían por primera vez hablar y cuestionar temas que, hasta ese entonces, eran desconocidos para ellos, acaso nunca problematizados antes:

"Reflexionando que es una actitud aprendida, que se "normaliza", que muchas veces tiene que ver con un miedo propio que se disfraza bajo esta actitud dominante sobre otros." (Audios de sesión grupal).

Entonces, comprendiendo desde los mismos relatos de los hombres que vivieron el proceso, que los aprendizajes traídos, respecto de una educación patriarcal y violenta, nunca antes habían sido cuestionados, y habían derivado en situaciones violentas contra ellos mismos $u$ otras personas, se nos hace inevitable pensar en la necesidad de que muchos, sino, todos los hombres tengan acceso a una educación y espacios de transformación, que permitan estas problematizaciones.

Sin embargo, las políticas públicas nacionales en este sentido, si bien hacen un trabajo en torno a la sanción como estrategia de prevención para que hombres no ataquen deliberadamente a las mujeres, apuntan a una intervención casi absolutamente con las mujeres víctimas de violencia. La actual 20.066 Ley de Violencia Intrafamiliar sigue estando supeditada a aspectos de violencia en donde la mujer mantiene una relación y/o vínculo de convivencia o legal con el hombre.

El total de hombres de esta investigación fueron derivados desde tribunales de garantía o de familia. Ninguno de ellos asistió de manera voluntaria. Dato no menor, ya que esto nos llevó a pensar y cuestionar ¿qué pasa con todos esos hombres, que al igual que los integrantes de nuestros grupos no tuvieron la oportunidad de encontrar un espacio en sus historias de vida, en donde fueran capaces o motivados a cuestionar-se- sus conductas y aprendizaje patriarcal?

Si los índices violencia hacia la mujer-y en general- aumentan, entonces ¿Por qué seguimos como país enfocados en aumentar la cantidad de centros para las mujeres víctimas de violencia y no enfocamos también recursos en la prevención y re-educación de los hombres, si son ellos quienes consuman femicidios y suicidios?

Los Centro de Hombres actualmente tienen cobertura regional, es decir, en la IV Región existe un solo centro, con un equipo profesional de tres personas (Un coordinador Psicólogo y una dupla Psicosocial, formada por un psicólogo y una trabajadora social) y una administrativa, para intervenir a toda la región que es derivada.

Resulta necesario y urgente el encontrar espacios en donde la población pueda recibir aprendizajes vinculados al desarrollo de la persona como un ser integral, como 
forma de resguardar el bienestar de mujeres, niños/niñas y jóvenes, y por supuesto, de los mismos hombres. Necesitamos urgentemente que la educación de todas y todos se base en llevarnos a pensar, cuestionarnos. De otra manera no estaremos interviniendo a las personas en riesgo sino cuando el daño ya esté hecho. Chile no es un país que se caracterice por las políticas y programas de prevención, y esta no es la excepción.

Como estrategia, quienes facilitábamos los grupos siempre participamos como uno o una más, relatando experiencias, hablando desde nuestro propio sentir, e incluso compartiendo lágrimas y risas. Esto generaba en los hombres una cercanía y sensación de confianza con quienes daban vida a la instancia. No era extraño para nosotros/as que nos comentaran que el centro de hombres era el lugar en donde se habían sentido escuchados por primera vez.

A su vez, se destaca el reconocimiento que los hombres comienzan a hacer en torno a relaciones basadas en el respeto y el miedo, reconociendo cómo estos elementos han forjado sus relaciones laborales y personales, y cómo fueron educados desde el miedo:

"logra realizar una fuerte crítica en cuanto al modelo educacional actual, reflexiona en torno a cómo es facilitador de conductas amenazantes y violentas, dentro de su espacio como profesor. Hace hincapié en alternativas que el mismo establecimiento le puede brindar frente a una situación por ejemplo de inquietud del alumno o alumna", "...por ejemplo, la mamá siempre tiene siempre la costumbre de decirle al hijo "ya va a llegar tu papá y vas a hablar con él”... está infundiéndose que el niño a mí me tenga miedo en vez de respeto...está infundiendo el miedo...y de ahí pasa que el hombre es el diablo de la casa... le decía siempre "va a llegar tu papá" ..."(Audios de sesión grupal).

Con la problematización de algunas de sus conductas, los hombres comienzan a generar empatía hacia el dolor que otras personas sufren:

“...me dejas entender que no solamente quien recibe el daño o quien sufre, sino también a mí me pasan cosas, consecuencias variadas, pero también pasan cosas a uno, más allá de los temas legales y todo lo que acarrea." (Audio de sesión grupal).

Reconocen los ejercicios de violencia y los problematizan, responsabilizándose. Además, hacen un trabajo en grupo de identificar y narrar situaciones personales con el fin de poder resolverlas en sesión, sin importar hace cuánto tiempo haya ocurrido desde aquella situación. En estas sesiones grupales, los hombres logran hacer una reflexión y crítica hacia las creencias machistas hegemónicas, principalmente las que discriminan la diversidad sexual, así como las actitudes violentas que los hombres han ejercido en relación de pareja con las mujeres:

"Darme cuenta de lo equivocado que estaba en mi forma de ver la vida en pareja, así como también lo que realmente tenía en mi entorno familiar, pude valorar realmente todo lo que rodeaba", "Se enseñaron técnicas para no llegar a la violencia verbal, ni física, las cuales aprendí satisfactoriamente, al punto de entender que las decisiones deben ser analizadas y tomadas junto a la pareja, que debe existir una previa conversación." (Audios de sesión grupal). 
Los hombres, a medida que avanzan temporalmente en el proceso, comienzan a identificar cambios, no sólo en su diario vivir (lo cual se corrobora en las entrevistas a sus parejas o ex parejas) sino, también, en el actuar presente en plenas sesiones grupales.

El modelo hegemónico de masculinidad limita la expresión de una amplia gama de emociones para los hombres (Kimmel, 2000), el espacio de trabajo grupal, por el contrario, les permite sentirse liberados (Lozano, 2017) para expresarse de formas alternativas a la que la masculinidad imperante les impone. Como señala Lozano (2017), los hombres aprenden que, para demostrar la posesión del control sobre las mujeres, no les importa si tienen que gritar, pegar, insultar, o de forma menos evidente, utilizar el silencio y la mirada para ejercer poder. Es más, rara vez se preguntan si existen otras formas de relacionarse con sus parejas o hijos/as.

Al generarse un espacio de convivencia respetuosa, una resolución de conflictos libres de violencia y de organización colectiva, dentro de los grupos de hombres en proceso de cambio, se practican nuevas formas de comunicarse y resolver conflictos que no tenían incorporadas:

"Antes era poco tolerante, explotaba, y ahora no...escucho más y tengo más paciencia..."; "ya no se acelera por cualquier cosa...ya no le echa la culpa a uno por cualquier cosa...como que antes él quería ganar a toda costa...se ha portado bien". (Reportes de la mujer).

Estas formas de comunicarse, paulatinamente las incorporan a su cotidianeidad, llevan a sus hogares y relaciones íntimas, y provocan cambios en los distintos subsistemas familiares, alterando las dinámicas conflictivas que se habían establecido e introduciendo puntos de fuga por donde generar el cambio en sus vidas:

"escuchar antes de actuar"; "ahora puedo conversar con personas que piensen distinto a mí". (Audios de sesión grupal).

Gran parte de la comprensión que los hombres van desarrollando, creemos que va de la mano con el enfoque de violencia que tenemos en este espacio educativo, que tiene como sustento teórico y conceptual a la "rueda de poder y control de Duluth".

Este instrumento entiende la violencia que ejercen los hombres contra las mujeres en las relaciones de pareja como un conjunto de comportamientos específicos y característicos. Si se observan en conjunto se logra identificar un patrón abusivo, que tiene una intención "Lo central de este modelo es que comprende la violencia masculina como un comportamiento con finalidad, y no como una reacción explosiva sin sentido. Se entiende que los comportamientos violentos tienen un propósito: el imponerse sobre la mujer, las ganas dominio sobre ella, y el controlar su forma de vivir, de pensar o de actuar" (SERNAMEG, 2011).

Uno de los elementos fundamentales del proceso con los hombres, fue el desarrollo de la empatía por el daño causado hacia sus parejas o ex parejas, e hijos/as. Entender lo que habían causado con sus conductas violentas y hacerse responsable de ello, les permitió comprender la magnitud de sus actos y el deber que tenían en cuanto a reparar aquel daño. 
La mayoría, al iniciar el proceso, buscaba una ganancia secundaria al asistir: desde evitar el término de su relación de pareja, hasta solventar problemas judiciales. Sin embargo, en la medida en que el proceso comienza a avanzar, tanto hombres como mujeres (sus parejas), señalan que esto comienza a cambiar, y los hombres del proceso se interesan más en saber de su compañera, cómo se siente y cómo vivió los ejercicios de violencia, así como hacer esfuerzos de reparar el daño causado,

"hay unos pequeños detalles y pedir disculpas porque uno también se equivoca, no es pura maravilla y uno se acuerda y pide disculpas, antes no po";: "enfrentar la situación incluso desde otro lado, como saber cómo piensa ella, igual facilita la interacción y la solución". (Audios de sesión grupal).

\section{Facilitadores y obstáculos en el proceso}

Un elemento que los hombres del proceso valoraron significativamente como facilitador de éste, es el respeto que se genera en el espacio grupal entre sus participantes. Se refiere a escuchar al otro/a, aceptar que tiene una opinión o experiencia distinta a la mía, responder sin ejercicios de violencia, incluso cuando se generen conflictos o tensiones en el grupo, y de la misma forma, participar de manera libre y activa, pero manteniendo el respeto por el espacio, la opinión y la integridad de la otra persona del grupo.

Es preciso señalar que estas instancias de respeto y escucha activa no se generan desde un inicio, sino, más bien, se desarrollan en la regulación misma que tanto el grupo, como quienes facilitamos las sesiones íbamos poniendo en juego: reglas explícitas de grupo (respetar la opinión del otro, no enunciar chistes o frases machistas), o implícitas (no señalar el nombre de los participantes fuera del espacio grupal).

La dinámica que se genera en grupos de hombres formados en una masculinidad hegemónica, tiende a tener como característica principal la competencia. Se privilegia la fuerza y la descalificación como formas de comunicarse, valorando con mayor seguridad y como personas más deseables quienes adoptan dichas características. En este espacio, a partir de reglas grupales establecidas desde las orientaciones técnicas (las cuales tienen que ver con "no juzgar al compañero"; "respetar la experiencia de los demás") se comenzó a generar un proceso grupal de horizontalización en el encuentro.

Paulatinamente, los/as participantes adquirieron otras formas de comunicación, de entablar relaciones en las que ni el ejercicio de poder sobre otros/as, ni la burla o la humillación, eran objetivos de la comunicación en sesiones grupales. Por el contrario, se generaba un espacio de solidaridad, en el cual los participantes se disponían a ayudar a sus otros compañeros a entender, reflexionar, ser confrontados o incluso representar sus experiencias de vida, para que, de manera colectiva, todos/as tuvieran la oportunidad de cambiar. En este sentido, los hombres mencionaban que esta comunicación se daba desde conversaciones informales entre compañeros:

“...justamente esas conversaciones informales son en donde se comparten cosas incluso privadas...donde hemos buscado inconsciente...una ayuda, una opinión...., “...yo 
como les digo, me afiancé...en los dos grupos... y creo que va en uno, y tratar de entenderse entre las distintas formas por lo que estamos acá", "la confianza que tenían de contar lo que les había pasado...y nosotros creer...de revisarlo, de los temas que hablan", "a medida que pasa el tiempo de los temas que íbamos hablando, íbamos conociendo más...", "también uno se suelta, como que aprende a confiar más en el otro" (Audio de sesión grupal).

Se genera un quiebre en la narrativa del hombre, teñida por la masculinidad hegemónica y sus mandatos, generando una fisura en donde ingresan otras experiencias de compartir con hombres y mujeres, otras formas de construir espacios horizontales en igualdad:

"uno de los ejercicios realizados, tenía como objetivo lograr la confianza en el compañero, una de las cosas a las cuales no estoy acostumbrado, pero fue muy gratificante y enriquecedor" (Audio de sesión grupal).

Junto con esto, los participantes identifican la forma en que los facilitadores nos comunicábamos, transmitiendo cierta forma de relacionarnos, generando un espacio de respeto y colaboración participativa:

"yo noto en ustedes, la calidez que tienen para decir las cosas, tratarnos igual como si nos hubiesen conocido hace mucho tiempo... a eso voy yo...en la forma como las dicen ustedes...como tratan de que nosotros entendamos a lo que ustedes quieren llegar." (Audios de sesión grupal).

Pero además de la confianza generada en el espacio grupal, los hombres destacaron la participación, como elemento activo del desarrollo colectivo:

"al escuchar a mis compañeros y sus historias...lo cual me ha servido para ver en lo que yo realmente estaba equivocado." (Registro de atención individual).

La apuesta educativa se construía de manera horizontal como un proceso de diálogo abierto, crítico y problematizador, en donde todos quienes participaban entraban a jugar un rol de facilitadores y protagonistas, en distintos momentos del proceso.

Aun estando insertos en un contexto sociocultural político de represión a la colectividad, el encuentro y la expresión emocional, los hombres comienzan a adquirir como propias algunas prácticas de resistencia ante dicho contexto ("escuchar lo que ustedes nos han enseñado, aprender de los temas, palabras que no conocíamos o quedábamos en la duda"). A pesar que todos llegan en situación de crisis vital (ruptura de pareja; alejamiento de su familia; sentimiento de culpa, otros), identifican que el espacio grupal conlleva elementos distintos a los que se encuentran acostumbrados a vivenciar en otros espacios.

Por lo tanto, comienzan identificando la acogida que se entrega en Centro de Hombres y la disposición a escucharlos y creer en ellos, como elementos de valor humano: 
“...es como apoyo que nos dan ustedes desde el principio, el poder estar aquí, de escucharnos. De decir: esto va a ser así, y el tiempo que se dan pa' estar aquí con nosotros y el valor humano." (Audios de sesión grupal).

De igual manera, los hombres del proceso grupal, reconocían que en otros espacios se encuentran con un tipo de relación más instrumental y superficial, muchas veces, teñida de violencia y competencia. Esto inevitablemente se encuentra ligado a que el aprender a escuchar activamente sin juzgar, les permitía problematizar su propia situación personal, observarse en el otro, mientras que el otro compañero propone su texto en el contexto grupal que acoge:

"a mis compañeros por darme la posibilidad de sanarme con ellos ya que me ayudan a entender que soy una persona que comete muchos errores...ya que como hombres necesitamos conocernos cada vez mejor a nosotros mismos." (Audio de sesión grupal).

Si bien en ciertas sesiones, los hombres generaban alianzas, unificándose en torno a discursos patriarcales, y éstas se convertían en tensiones importantes frente a las temáticas a trabajar (culpar a la mujer, generalizar las experiencias), de manera paulatina, y a través de la resolución colectiva de dichas tensiones en el mismo espacio de sesión grupal, las formas de expresarse se tornaban menos sexistas. Es decir, no se estaba replicando nuevamente un grupo machista, sino más bien, los hombres, al identificar estas formas de acoger y comunicar, transformaban la manera de hacer grupo, de hacer colectividad entre hombres. Como señala Kaufmann (1994), la alienación de los hombres se relaciona con la incapacidad de reconocer emociones, sentimientos y necesidades del potencial para relacionar su humanidad con los cuidados. Esta alienación también resulta de la distancia de los varones con las mujeres y de la distancia y aislamiento con otros hombres. Este aislamiento es la clave para conservar el patriarcado.

En estos espacios grupales construidos en HEVPA, la distancia entre nosotros/as cada vez fue menor en la medida en que nos relacionábamos de otras formas.

Respecto a los obstaculizadores del proceso, los hombres identifican la "vergüenza" por estar en un centro de hombres, esto, desde la ignorancia del trabajo en masculinidades y desde la escasa difusión de la temática:

"Antes como que uno llegaba y le daba vergüenza hasta golpear la puerta, como que uno igual empezaba duro. Me vas a creer que las primeras veces que venía para acá había un niño que lavaba autos y me miraba y yo lo miraba y me daba como un poco de vergüenza y ahora ya no, aprendí eso ya no tengo miedo, cachai, que eso pasaba.", "la vergüenza porque es mal mirado como nosotros estamos en un centro de hombres y ejercimos violencia nos miran mal desde afuera y nosotros transformamos esa vergüenza en virtud." (Audios de sesión grupal).

Que ellos buscaran y visualizaran el centro como un espacio de contención y reparación es evidencia de que no existen lugares para que los hombres puedan expresarse y trabajar sus emociones y violencias. En trabajo grupal, por ejemplo, se evidenciaba que desde la propia "masculinidad" de cada hombre, estos ya rechazaban la 
posibilidad de mostrarse emocionados ante otras personas, sobre todo, otros hombres, y junto con esto, las actividades que tenían relación con el contacto físico o demostración de afectos a los compañeros comenzaban con risas o incomodidad entre ellos.

A medida que avanzaban las sesiones grupales, aparecen aspectos que tienen relación con el sentimiento de vergüenza, pero esta vez, por los actos cometidos hacia las personas más significativas para los hombres:

"Dejar de lado la vergüenza es un trabajo intenso, poder contar tus cosas intimas a otras personas, el reconocer que cometiste errores frente a algún desconocido en otro momento." (Audio de sesión grupal).

El reconocerse como un hombre que violentó a una mujer es parte de esa vergüenza que sienten, se vuelven más conscientes de que sus violencias no fueron actos sin sentido, sino que tuvieron consecuencias reales, para ellos, ellas y sus hijos/as.

En Chile existe un Centro de Hombres por región, y las distancias de los alrededores cobran importancia en la adherencia del hombre al proceso. Por lo mismo, la mayor cantidad de hombres asisten desde la ciudad de La Serena (donde se encuentra ubicado el centro) o desde la ciudad de Coquimbo, desde la cual se pueden transportar sin gran dificultad. Caso contrario presentan los hombres que residen en ciudades más distantes, en donde el transporte involucra un gasto económico y el tiempo de traslado resulta ser lapidario para la regularidad que el proceso exige (una a dos sesiones mensuales en etapa individual; una sesión semanal en etapa grupal). En grupo de segundo nivel (etapa más avanzada) los aspectos que reconocen como obstaculizadores para su proceso son: el contexto en donde se desenvuelven en términos relacionales (es decir, si sus parejas, espacios laborales o familia criticaban sus cambios en las conductas como desviaciones de "lo que un hombre debe hacer"), y un "miedo y temor" al proceso, desde el desconocimiento de lo que podrían "decirle" o se podría "hacer" en este espacio:

"No saber, a lo que estábamos...a lo que nos podíamos enfrentar, a lo que venía. A lo que se hace acá. El temor a lo que pueda suceder. Uno cuando llega es como huaso en el mall" (Audios de sesión grupal).

Los hombres, generaron un cuestionamiento a la real efectividad de su intervención en ese momento, comparándose con quienes no lo estaban viviendo:

"La gente que no vive este proceso y que se relaciona con nosotros". (Audios de sesión grupal).

Señalaban que resultaba complejo relacionarse con personas "fuera" del centro, debido a que mantienen y reproducen creencias y acciones machistas que, a diferencia de ellos, lograban ver y problematizar. Consideramos muy relevante este elemento destacado por los hombres, ya que, por una parte, comienzan a identificar al resto de la sociedad que presenta elementos que califican de "machistas", como otro, que no accede a -en palabras de ellos- "el privilegio de pasar por un proceso como éste". Junto con eso, le añaden la característica de ser otro "peligroso" y un obstáculo para su proceso de cambio, ya que ellos sienten que son alentados por este grupo de personas a re-producir conductas violentas y machistas, que ellos intentan abandonar. 
En contraposición a esto, es preciso explicitar que los distintos tribunales que derivan a los hombres no ponen la misma atención a sus causas, no siempre existe un seguimiento por el riesgo que la víctima puede presentar. Por tanto, los informes que se solicitan de dicho espacio (principalmente, desde tribunal de garantía ocurre esto) se requieren cuando se cumple cierto plazo de las medidas sugeridas al hombre, sin embargo, la víctima queda en el mismo nivel de riesgo, más aún cuando el hombre no reconoce la violencia ejercida. Por tanto, cumple con uno de los criterios de no calificación a este espacio: "que el hombre no reconozca ningún ejercicio de violencia contra la pareja o ex pareja”. Entonces, nos preguntamos, ¿Qué tanta incidencia tiene las medidas y protocolos o procedimientos penales en cuanto a reproducir y mantener la impunidad de hombres agresores y el riesgo de las víctimas?

Hace un poco más de 20 años atrás ya existía evidencia de la importancia y urgencia de considerar a los sistemas que se relacionan con la persona que ejerce la violencia y sus víctimas. Lo explica Ravazzola (1997) describiendo tres actores claves que interactúan como un circuito cerrado que se perpetúa a sí mismo: la persona abusadora, es quien ejerce violencia, la persona abusada o violentada, que generalmente son mujeres, niñas o niños, ancianas o ancianos, o cualquier persona en situación de opresión; y por último, las personas testigos o que son parte del contexto. En este caso, entendemos al sistema judicial, como un dispositivo de poder, que si bien, plantea cierta fuerza punitiva a los ejercicios de violencia en la relación de pareja, a su vez, perpetúa un nivel de impunidad, al adoptar prácticas negligentes y re-productivas del mismo sistema de opresiones, como ordenar al hombre que acuda a un proceso psicológico para "sanarse", pero no haciéndose cargo de los aspectos cualitativos de dicho proceso, ni muchas veces, prestando la atención necesaria a ello. Es decir, en la mayor parte de los casos, la preocupación de tribunales recalaba en la sola información de si el hombre dio cumplimiento con la orden emitida, es decir, si acudió o no a este espacio. Es por ello, que como señala Ravazzola (1997) y SERNAMEG (2019), en sus lineamientos, la respuesta para detener la violencia masculina debe ser comunitaria. Pero mientras se mantengan dispositivos que ejercen cierto poder (por solo poner este ejemplo, el poder judicial), que no se logren alinear con el enfoque del resto de los niveles de respuesta comunitaria (centros de atención psicosocial, familias, comunidades barriales, entre otros), no será posible tener una incidencia tan efectiva como se espera en la detención de la violencia.

\section{Lenguaje, discursos y violencia}

Se evidencia que mientras los hombres se encuentran atravesando la etapa grupal de primer nivel, no tienen una problematización de los ejercicios de violencia cometidos hacia sus parejas o ex parejas. Es decir, en este nivel, no hay evidencia de que vean la violencia como un problema del cual han sido responsables.

Los hombres en este espacio dejan claro desde un comienzo que la violencia, sea cual sea su forma y su destinatario/a, es algo que no se debe hacer. Efectivamente, existe cierta contradicción en sus relatos, al señalar que la violencia es una conducta 
condenable en nuestra sociedad, mientras argumentan que la mujer realiza acciones provocadoras de la violencia hacia ellas. Un hombre que pasó por este proceso, entrevistado por Santibáñez y Portilla (2017), señala:

"vuelvo a insistir no son excusas cachay, pero siempre dejan mal al hombre, pero tienen que ver que detrás de esa persona pueden haber muchos dramas, como lo que me pasó a mí, me estaban gatillando los celos, porque yo creo que la mujer no respeta, o el hombre no respeta, según quien cometa el error, pero no se respeta, si la mujer fuera más honesta diría, sabí no me gustai, o sabí, no estamos bien ¿pero por qué ser infiel si tu escogiste una pareja? Porque yo he visto que en los casos que han matado a las mujeres, han sido casi siempre por infidelidad".

Si bien él recién estaba en la etapa individual antes de acceder al grupo, deja una muestra clara de cómo los hombres ponen sus creencias sexistas en juego al momento de entregar una comprensión de las situaciones de conflicto a nivel de pareja.

Para realizar ese trabajo de desmadejar capitalismo y patriarcado entreverados en un cuerpo mutilado de hombre que mutila a otros, debimos ser estratégicos/as. Observar todo lo que "nos traía" este hombre, desde su historia personal, su autobiografía, sus creencias, emociones, así como observarnos a nosotros/as.

Se puede pensar entonces, que recién se encontraban en un proceso de "llamar violencia a la violencia”, como señaló uno de los hombres del proceso grupal. Entonces, el lenguaje como constructor de realidades, recién en esta etapa, les permitió entender en qué situaciones habían ejercido violencia y por qué llamar violencia a algo que para ellos era normal.

En grupo de segundo nivel y hasta el egreso del proceso, se evidencia una problematización de las conductas machistas en el actuar personal de los hombres que pasan por el proceso, un desarrollo de la empatía por lo que su pareja o ex pareja pudo haber vivido, y el emerger de la necesidad de reparar el daño causado.

Entonces, mediante dinámicas y actividades diversas (psicodrama, juegos de roles, esculturas corporales, imaginerías, juegos, otros), la conciencia de las situaciones tiende a ampliarse más allá del nivel individual. Son capaces de conectar con las otras dimensiones que conllevan su experiencia de haber ejercido violencia y, por ende, entender no sólo desde otros puntos de vista la situación, sino que lograr una comprensión crítica de su experiencia.

El hombre que está en proceso grupal se hace cargo de sus emociones, las logra visibilizar, por lo tanto, también comienza a ver a su pareja o ex pareja como alguien que es capaz de sufrir, y dejar de verla solamente como una enemiga o una amenaza:

"Recuerdo cuando creíamos en nosotros, era tan robusto solo teníamos amor, a medida que fue pasando el tiempo algo en mi cambió, no supe frenar mis impulsos, mis actitudes de machista fueron aflorando cada vez con mayor violencia al punto de llegar a los golpes, siento una angustia inmensa al recordar tu rostro en ese momento." (Registro de atención individual). 
Surgen nuevas palabras para categorizar lo que alguna vez hizo, y, por lo tanto, la comprensión de lo que vivió en el pasado él y su pareja. Comienzan a entrar en juego elementos que hablan de cómo se sentía (angustia, pena); se empiezan a explicar cuáles fueron los orígenes de su actuar y a responsabilizarse de sus actos en vez de culpar a la pareja.

Para Freire, la concientización es un elemento inseparable de la liberación. Chesney señala: "la concientización es la mirada más crítica posible de la realidad, y que la desvela para conocerla y conocer los mitos que engañan y que ayudan a mantener la realidad de la estructura dominante". (Chesney, 2008: 54).

En el caso de los hombres en grupo, si bien intentábamos des-velar esta realidad para observarla de manera crítica y des-armarla, des-montarla, también, durante las conversaciones informales o en las mismas discusiones y reflexiones grupales, aparecían críticas hacia el sistema de gobierno, el sistema económico y cómo ello impactaba en la vida de cada uno de nosotros/as. Como era de esperarse, terminábamos vinculando patriarcado y capitalismo de una manera muy espontánea. Por tanto, los hombres de manera personal desarrollaban esta conciencia de sus actos y las consecuencias, y a la vez, de cómo empezar a des-armar, para construir un nuevo hombre. Esto, lo traducían en compromisos, que iban principalmente dirigidos hacia su pareja o ex pareja:

"me comprometo a ponerme en tu lugar, me comprometo a respetarte en todos los sentidos, me comprometo a ser verdadero, me comprometo a apoyarte en todos tus sueños, me comprometo borrar todo lo oscuro que viviste a mi lado, me comprometo a que seas feliz." (Registros de atención individual).

Uno de los elementos que más fácil resulta cambiar es el discurso. Entonces, si bien siempre se les sugería a los hombres en grupo que fueran lo más sinceros/as posibles, sucedía que luego, cuando realizábamos las entrevistas a las parejas o ex parejas, algunos continuaban ejerciendo violencia, a pesar de haberlo negado en espacio grupal.

Puede existir concientización de la violencia y de la realidad como hombre en una sociedad patriarcal, pero eso no quita que pueda continuar ejerciendo poder y control a pesar de su conciencia de ello y de sus consecuencias. Ahí surgen entonces nuevas preguntas: ¿Qué lleva a los hombres a mantener ciertas conductas a pesar de haber concientizado la violencia ejercida?

Si bien permitíamos que se expresaran respetuosamente creencias respecto a la mujer (muchas veces culpabilizándola o juzgándola), debíamos mantener como facilitadores una postura política (ante la desigualdad y opresión) y una mirada desde la educación popular, para alentar la concientización del patriarcado inmerso en sus creencias.

Korol lo señala claramente: "una praxis feminista implica poner nuestra acción al servicio de no reproducir ni que se reproduzcan estas situaciones de subordinación en el ámbito en que nos toca desenvolvernos" (Korol, 2006: 59). Si alguien se declara profeminista, o feminista, al servicio de detener la reproducción de la opresión y las 
desigualdades, el elemento determinante es realizar una praxis cotidiana. En este espacio grupal hacíamos el esfuerzo máximo por minimizar los ejercicios de poder, desde todos los roles que jugábamos. Korol (2006) termina su idea señalando que esto exige estar atentos/as en nuestra vida cotidiana, como lo fue la posición feminista que sostuvimos, y revisar en el cotidiano como facilitadores, porque, aunque muchas veces los hombres nos veían como "los que saben", inevitablemente el patriarcado y el capitalismo nos atravesaban. A medida que se abrían plenarios respecto a los temas o a los materiales audiovisuales revisados, aparecían recuerdos de la crianza de cada uno de nosotros/as, y también, de espacios extrafamiliares que nos transmitieron en algún momento, la violencia como forma de resolución:

"el que ejerce la amenaza, siempre va a conseguir algo, conseguir algo que con el dialogo normal cree que no lo va a hacer...acude a la amenaza para conseguir lo que quiere..." (Audios de sesión grupal).

Por último, los hombres señalan en sus discursos que logran determinar que la violencia es un elemento inaceptable en esta sociedad. Dentro de sus discursos, se evidencia cierta justificación hacia las violencias que las mujeres sufren, así como también las situaciones ocurridas con sus parejas. El lenguaje adquirido por los hombres para nombrar la conducta violenta se torna además una herramienta para adaptarse y manipular su entorno, de modo que no se sufra una exclusión (del grupo, de la sociedad) por exponer sus ideas. Por ello, uno de los elementos que nos quedan al revisar la problematización grupal de los hombres, como señalaba Korol (2006) es un deber de observar constantemente, mantener una postura de reflexión y atención a lo cotidiano y a los discursos ahí producidos, a las acciones y omisiones. Como señala Connell (2003), la masculinidad es producto de un marco socio-cultural-histórico amplio, que determina los márgenes en donde se puede mover el discurso y las acciones de un hombre, pero además, lo tiñe de contradicciones, de manera que, por una parte, puede expresar una total deconstrucción y análisis de su masculinidad, y sin embargo, continuar ejerciendo control y violencia sobre su compañera.

\section{Problematización desde la vivencia de los hijos/as}

Un elemento significativo que nos llamó la atención, transversal en el relato de gran parte de los hombres, fue que comenzaron a problematizar y detener la violencia a raíz de haber observado las consecuencias de ésta sobre sus parejas, en sus hijos e hijas:

"Yo me quise ir de ahí para evitar discutir frente a mi hija; no me deja ir, me sentí encerrado asustado e impotente, comencé a gritarle, ella también lo hacía, estábamos fuera de sí, no recuerdo si quiera lo que le dije, pero supongo que fue lo más hiriente que mi cerebro pudo elaborar. Cuando vi a mi hija llorando se me enfrió el pecho y salí de ahí como pude", "Después de un rato reaccioné al escuchar los llantos de mis hijos." (Registros de atención individual). 
Sólo cuando los hijos/as han estado presentes en las escenas de violencia, los hombres logran reconocer que su violencia tuvo consecuencias. Antes de esto, no problematizan desde el dolor de su pareja. Aquel castigo ejercido hacia ellas está justificado en su discurso por diversas creencias, que transitan desde el merecimiento de éste, hasta la culpabilización hacia ellas por "haberlos provocado".

Vale la pena entonces preguntarse, ¿cómo influye el que ellos observen a sus hijos ser testigos de la violencia?, ¿cómo los impacta el ser observados por sus hijos, al punto de problematizar la situación de violencia contra la pareja?

\section{Vivencia de la mujer}

Una de las formas de contrastar los discursos de los hombres fue entrevistar a la mujer, pareja o ex pareja de ellos. Este relato podía ser proporcionado por los Centros de la Mujer, o bien cuando algún miembro del equipo tomaba contacto con ella.

Se evidenció en sus relatos que las mujeres esperaban cambios inmediatos en las conductas de los hombres al momento de ingresar al proceso, pero los hombres continuaban ejerciendo privilegio masculino (en la crianza), externalizando la responsabilidad a las mujeres; mantenían el uso de los niños y niñas para manipular y controlar a la mujer, principalmente en quienes estaban separados y; abuso económico (no pago de pensión alimenticia).

Por ejemplo, las mujeres reportan:

"el cumple con la pensión y las visitas, pero yo me llevo el peso, él se excusa por trabajo, a veces le pido...pero se excusa... no le ayuda en las tareas, disciplina...él también tiene que ser participe...", "No veo interés...no hace tareas...no la baña... aparece a la hora que le da la gana...habla por medio de la mamá...no sé por qué es un problema de él".

El abuso económico por parte de los hombres se mantiene, y es algo que pocas veces reconocen, llevando a las mujeres, a generar ingresos por diversos medios y normalizando el hecho de que ellos no cumplan con dicha responsabilidad:

"lo que él promete no lo cumple... antes tenía más cercanía con la niña...tampoco la pensión...yo tengo que estudiar, que trabajar y hacerme cargo de la niña...si tenemos que llegar al juicio lo voy a hacer..." (Reporte mujer).

Otra mujer, señala:

"Respecto a lo económico, él adeuda $\$ 3.590 .000$ en concepto de alimentos respecto a la hija, teniendo una orden de arresto por no haberse presentado a reclusión nocturna." (Reporte mujer).

Quienes cumplen en lo económico, comienzan a dejar de lado otras responsabilidades y comienzan a ejercer privilegio masculino, al no velar por las labores que se deben realizar dentro del hogar y en la crianza de los hijos/as. Las visitas que tienen los hombres con sus hijos/as, cuando están separados de la madre, siguen siendo 
espacios en donde la manipulación de los niños/as se mantiene. Muchas veces estos son utilizados para obtener información de la madre, emitir juicios sobre ella:

"ha hecho comentarios na' que ver... pero prefiero no tomarlos en cuenta, mi hija llega contando que a veces dice cosas de la mamá...como que es vieja." (Reporte de la mujer).

Por otra parte, las mujeres reportan que el consumo de alcohol en los hombres disminuyó mientras ellos estaban en el centro:

"Ya no toma en la casa, antes él quería hacer un asado y el hijo le decía yo voy a comprar algo y se armaba la discusión, por ejemplo, eso no pasa...", "él no toma ahora casi nada, y antes hacía asados y se convertía en otra cosa, insultos, peleas... cuando tomaba, como que se pierde..." (Reporte de la mujer).

Durante el tiempo que trabajamos con ellos, las orientaciones técnicas del centro recomendaban una forma distinta de trabajar las temáticas de segundo nivel. Esta forma decantaba por un trabajo emocional y dirigido hacia otro tipo de masculinidades, más quecomo se trabajó en primer nivel- sobre el reconocimiento y la problematización de los ejercicios de violencia cometidos. Si bien cada actividad que permita al hombre reflexionar sobre su identidad masculina trae consecuencias positivas tanto para su salud como para sus relaciones, creemos que el disminuir la directividad y la especificidad de las temáticas a trabajar, trajo resultados positivos, pero, además, permitió que ciertas violencias se mantuvieran ejerciendo en el espacio íntimo de la relación de pareja:

"yo no lo veo desde mayo, que vino para el cumpleaños de mi hijo...personalmente no...solo por celular...me bloqueó de WhatsApp...solo por correo... ahora a fines de septiembre recién cambió...me mandaba correos diciéndome de todo..." (Reporte de la mujer).

Se evidencia también un daño sistemático e histórico en la mujer, recibido durante la relación de pareja, el cual muchas veces debió recibir atención de salud mental:

"Siento miedo que pase el tiempo y todo siga igual. Él no me escucha y ahora me escucha un poco más, por lo menos me pone atención, siento que lo que sale de mi boca no es importante... me estoy sanando yo que estaba herida", "y antes la respuesta de él era 'yo no estoy con ustedes, tú tienes quien te apoye, haciendo mención a mis padres...ahora me va a ayudar con el colegio de los niños y el fútbol de mi hijo...yo me había quedado sin trabajo y no teníamos para comer, estuve muy mal, me daban crisis, estuve en tratamiento con psiquiatra..." (Reporte de la mujer).

De acuerdo con las entrevistas hechas a las mujeres, la violencia física se logra detener con el modelo de intervención presentado; las otras manifestaciones se mantienen, considerando que estas mismas son replicadas por sus mismos círculos y por la sociedad misma:

"Refiere que han logrado llegar a acuerdos en la crianza de sus hijos, compartiendo tareas y ha cumplido con la pensión de alimentos", "Todos notan el cambio, 
su familia, las vecinas", "Después ya no sigue en la pelea...antes seguía al otro día." (Reporte de la mujer).

\section{¿Qué nos quedó de lo vivido?}

Fue fundamental que los hombres, en etapa grupal, experimentaran un acercamiento progresivo en torno a los ejercicios de violencia, así como considerar en la intervención que hay un contexto histórico y biográfico tanto personal como colectivo. Conocer el concepto de violencia: tipos de ésta, ejemplos y consecuencias; luego, cuándo y cómo la aprendieron; para entonces, recién comenzar a profundizar en las vivencias de pareja. Los hombres que comienzan el proceso tienen habilidades comunicacionales notoriamente disminuidas, por tanto, el espacio grupal se presenta como un lugar idóneo para desarrollarlo. Se destaca que lograron reconocer cómo se educa en torno a la violencia en distintos espacios: entendieron que desde que nacemos adquirimos un aprendizaje sexista, patriarcal y violento. Por tanto, creemos necesario y urgente generar espacios desde la educación pre-básica que les permita a los hombres dejar de lado el analfabetismo emocional, y re-educarse en otros ámbitos ajenos a la productividad y lo técnico, y más centrados en el propio cuidado y cuidado de otros/as.

En cuanto a la metodología de trabajo grupal, existió una reticencia de los hombres a probar nuevas formas de relacionarse corporalmente (con sí mismos y con otros) en el espacio grupal. Entonces creemos importante la necesidad de considerar al cuerpo como una dimensión fundamental de trabajo con hombres ya que por éste entendemos que atraviesa no sólo el patriarcado, sino que también el capitalismo.

El espacio grupal por su parte fue un potenciador de la participación y resolución de conflictos personales y entre los mismos participantes.

Creemos entonces que es fundamental cuestionar y re-significar constantemente el rol que cumplimos en distintos momentos de un proceso de cambio relacionado con la violencia en sus distintos espacios y manifestaciones, en oposición a volvernos un obrero/a social que sólo obedece y reproduce, y no se pregunta ¿Para quién? ni ¿Para qué?, Sino que proponemos poner el cuerpo y la vivencia personal de quien facilita un proceso como éste, al servicio de una transformación grupal y comunitaria en contraposición a ejercer un rol controlador y autoritario.

Se evidencia un alto nivel de cambio en los discursos de los hombres, incluyendo elementos de igualdad y de resistencia al patriarcado, sin embargo, la violencia se mantiene en la relación con la pareja y/o ex pareja. Ante esto, una de las praxis que destacamos fue el contacto constante con las mujeres, no sólo para enfocarnos en resguardar su seguridad, sino también, su relato en primera persona como retroalimentación principal del proceso del hombre. Es fundamental que además de proponer estrategias para resguardar la protección de la víctima y su proceso de reparación, existan alternativas, espacios para que los hombres cuestionen sus violencias, trabajen sus dolores y aprendan formas resolutivas libres de violencia con tal de prevenir que ejerzan violencia hacia las mujeres. 
Si algo podemos concluir firmemente es que, si queremos cambios reales en torno a la violencia, género y masculinidades, es preciso que el rol que tenemos como educadores/as y actores/actrices en distintos espacios pedagógicos y dispositivos psicosociales(como señala Agamben, 2011), dispositivos estratégicos que resultan del cruzamiento de relaciones de poder y de saber, deba entonces tener un posicionamiento crítico y político, en constante revisión, y a su vez, ser estratégico, si por ejemplo, como en nuestro caso, buscábamos visibilizar elementos transformadores respecto a las relaciones de poder. Por nuestra parte, por último, interpelamos a los hombres a generar instancias de fisura, de quiebre con este sistema patriarcal y capitalista, que nos permita entonces, construir de manera comunitaria, espacios e instancias de transformación a distintos niveles, que no dependan de respuestas del Estado y que consideren no solo la punitividad, sino, la responsabilización y el apoyo mutuo.

\section{Bibliografía}

Agamben, G (2011): “Qué es un dispositivo?” en Sociológica. 2011, Vol.26, n.73, p249264

Connell, R. (2003): Masculinidades. México DF, México. Universidad Nacional Autónoma de México.

Chesney, L. (2008): "La concientización de Paulo Freire" en Historia de la Educación Colombiana. No.11, p. 51-72.

Federici, S. (2017, 4 de agosto: "Ni caza de brujas, ni brujas en las casas", Entrevista de Silvia Federici con el medio "Zur". Recuperado de https://www.lahaine.org/mundo.php/video-federici-ni-caza-de.

Heise, L. (1998): "Violence against women: and integrated, ecological framework" en Violence Against Woman.Vol 4, No 3, p. 262-90

Kaufmann, M. (1994): Theorizing Masculinities. Newbury Park. Sage Publications.

Kimmel, M. (2000): The gendered society. New York: Oxford University Press.

Korol, C. (2006): Hacia una pedagogía feminista. Buenos Aires, Argentina. Editorial El Colectivo.

Lozano, I. (2017): Debates y Reflexiones en torno a las masculinidades: analizando los caminos hacia la igualdad de género. México DF, México. Facultad de Psicología UNAM. 
Revista Punto Género N.ำ 13. Junio de 2020

ISSN 0719-0417 / 4 - 24

Pescador, E. (2018): Cambio de las masculinidades desde la educación. Blog: Voces de hombres por la igualdad. Recuperado de https://vocesdehombres.wordpress.com/cambio-de-las-masculinidades-desdela-educacion/

Ravazzola, M. (1997): Historias infames: los maltratos en las relaciones. Buenos Aires, Argentina. Paidos.

Ríos O. (2015): "Nuevas masculinidades y educación liberadora" en Intangible Capital. Vol11, No. 3, p. 485-507.

Santibáñez, B. \& Portilla, J. (2017): "Estudio descriptivo acerca de la violencia de género dentro de una relación de pareja o ex pareja en hombres que acuden al centro "Por una vida sin violencia". Tesis (psicología). La Serena: Universidad Central.

SERNAMEG (2011). Boletín mensual º4. Unidad de Prevención de VIF Chile Acoge.

SERNAMEG (2017). Documento interno.

SERNAMEG (2019). Violencia contra las mujeres. Chile. Recuperado de https://www.sernameg.gob.cl/?page id=26815 